\title{
The T-Mixture Model Approach for Radar HRRP Target Recognition
}

\author{
Caiyun Wang and Jiling Xie
}

\begin{abstract}
This paper addresses the problem of automatic radar target recognition using high-resolution range profile (HRRP). We develop a t-mixture model (TMM) to model radar echoes from each target as the t-distribution forms. Estimation of the model parameters is achieved using a modified expectation-maximization (EM), i.e., fuzzy EM (FEM) algorithm. Numerical simulation results show that the proposed approach can achieve good performance of robustness and higher recognition rate. It is helpful for real-time identification and the engineering implements of automatic target recognition using HRRP.
\end{abstract}

Index Terms-High-resolution range profile (HRRP), radar automatic target recognition (RATR), t-mixture model (TMM), statistical model, expectation-maximization (EM).

\section{INTRODUCTION}

Radar High Resolution Range Profiles (HRRP) can be seen as the one-dimensional projection result of the electromagnetic scattering characteristics of the target as a function of the range along the radar Line-of-sight (LOS). It reflects the geometrical shape and structural characteristics of the numbers of strong scattering centers the relative position of scattering centers and the relative magnitude of scattering strength included in the target. Recently HRRP has been used as major signatures for radar automatic target recognition (RATR) [1]-[13].

From the view of signal representation, HRRP recognition methods can be roughly classified into two categories: methods directly using range profiles as classification features [1]; methods based on target features extracted from range profiles [2], [3]. References [4], [5] studied some time-shift invariant features of HRRP; [6], [7] discussed the variation of recognition performance with signal bandwidth; some literature showed that statistical recognition is an efficient method for RATR [8]-[11]. Beckman [9] proposed a model based on beta distribution, Gaussian model [8], Gamma model [10] and the two-distribution compounded model [11] was also proposed for HRRP samples. But the performance of existing statistical models is unsatisfactory in target identification. Beta model [9] is sensitive to target aspect. Thought the use of Gamma component distribution [10] arises from physical consideration, this model has computational reasons. The two-distribution compounded

Manuscript received January 10, 2013; revised April 23, 2013. This work was partially supported by the Youth Science and Technology Innovation Foundation of the Ministry of Education (NS2010221).

Caiyun Wang is with the Nanjing University of Aeronautics and Astronautics, Nanjing 210016 China (e-mail: wangcaiyun@ nuaa.edu.cn).

Jiling Xie is with the Nanjing Research Institute of Electronics Technology, Nanjing 210039 China (e-mail: xiejiling@sina.com). model [11] comprising Gamma distribution and Gaussian mixture distribution is a complicated one.

Multivariate Gaussian components for mixtures are ubiquitously focused on due to their analytical tractability, asymptotic properties and computational convenience. However, as stated by Peel and Shoham [12]-[13], the mixtures of Gaussians lack robustness in the presence of the observations that are atypical of the components, and therefore the fitting of mixtures of t-distributions is usually adopted alternatively as a standard choice. The t-distribution has a wider tail than the Gaussian distribution, and is immune from atypical observations of a component by giving reduced weight in the calculation of its parameters.

An Expectation-Maximization (EM) algorithm for mixtures of multivariate $\mathrm{t}$ - distributions algorithm gives a general solution for parameter estimation [14]. Unfortunately, traditional EM algorithm leads to poor parameter estimation when a single run of EM is used, for suboptimal solutions. Fuzzy C-Means method has a tendency to recommend solutions that equalize cluster populations. Therefore, we use the fuzzy expectation-maximization (FEM) algorithm to estimate the $\mathrm{t}$-distribution mixture model parameters.

In this paper, we consider multivariate t-mixture model (TMM) for target HRRP. Parameter estimation of t-mixture distribution is achieved by using the FEM algorithm. In the recognition experiment based on simulated data, the proposed statistical model not only has better recognition performance but also is more robust to noises than other methods.

The rest of the paper is organized as follows. In Section II, the TMM statistical model approach to HRRP-based statistical recognition is proposed. Section III introduces the modified EM algorithm, i.e., FEM used to estimate the parameters of the mixture model. The results from an application to radar HRRP target recognition are given in Section IV, and compared with GMM techniques. Finally, we present our conclusions in Section V.

\section{Statistical Model For TARgET HRRP}

\section{A. Introduction to Mixture Models}

According to Bayes theorem, we have $P(c \mid \mathbf{x}) \propto$ $p(\mathbf{x} \mid c) P(c)$, where $p(\mathbf{x} \mid c)$ is the class-conditional probability density and $P(c)$ is the prior class probability. Thus, estimation of the posterior probability is turned into estimation of the class-conditional density. For an HRRP including many range cells, nonparametric methods of density estimation, e.g., Parzen window method, are impractical due to the huge storage requirement. An 
alternative approach is to trade flexibility for robustness and to use some simple parametric form for target probability density. Mixture models [15] provide a convenient semi-parametric method for density estimation, and have been used in some form or another in numerous areas, including target tracking, speech recognition.

The general form of a mixture model is given by the following distribution

$$
p(\mathbf{x} \mid \Theta)=\sum_{m=1}^{M} \pi_{m} p_{m}\left(\mathbf{x} \mid \boldsymbol{\theta}_{m}\right)
$$

with

$$
\sum_{m=1}^{M} \pi_{m}=1,\left(0 \leq \pi_{m} \leq 1\right)
$$

where $\Theta=\left\{\pi_{\mathrm{m}}, \theta_{\mathrm{m}}\right\},(\mathrm{m}=1,2, \cdots \mathrm{M})$ denotes the parameter set. $\pi_{\mathrm{m}}$ are the mixing probabilities, and $p_{\mathrm{m}}\left(\mathbf{x} \mid \theta_{\mathrm{m}}\right)$ the parameterized component densities.

Given parameterized forms for the component densities, there are many techniques for estimating the parameters $\theta_{\mathrm{m}}$. The most common is the Expectation-Maximization (EM) algorithm, which estimates the maximum likelihood parameters. EM algorithm has attracted a great deal of interest over the past few years in a wide range of application involving tasks with incomplete data sets [16]-[17].

\section{B. T-Mixture Component of Target HRRP}

It is a great challenge in HRRP-based statistical recognition to give the accurate description of HRRPs' statistical characteristics. Typically, for continuous densities, the components are Gaussian and Gamma. As mentioned in the "Introduction" section, it is more reasonable to view the atypical observations from a t-mixture model (TMM). In the TMM, it is assumed that the data are from several components with distinct $\mathrm{t}$-distributions. In this work we use t-distributions.

Suppose $\left\{x_{1}, x_{2}, \cdots x_{N}\right\}$ are $D$-dimensional random observations of size $k$ and are generated independently from a mixture of $M$ underlying populations with unknown proportion

$$
p\left(\mathbf{x}_{k} \mid \Psi\right)=\sum_{m=1}^{M} \pi_{m} p_{D}\left(\mathbf{x}_{k} ; \boldsymbol{\mu}_{m}, \boldsymbol{\Sigma}_{m}, v_{m}\right)
$$

with

$$
\begin{aligned}
& p_{D}\left(\mathbf{x}_{k} ; \boldsymbol{\mu}_{m}, \boldsymbol{\Sigma}_{m}, v_{m}\right)= \frac{\Gamma\left(\frac{v_{m}+D}{2}\right)|\boldsymbol{\Sigma}|^{-1 / 2}}{\left(\pi v_{m}\right)^{D / 2} \Gamma\left(\frac{v_{m}}{2}\right)} \\
& \times\left(1+\frac{\delta\left(\mathbf{x}_{k}, \boldsymbol{\mu}_{m} ; \boldsymbol{\Sigma}_{m}\right)}{v_{m}}\right)^{-\left(v_{m}+D\right) / 2} \\
& \boldsymbol{\psi}_{m}=\left\{\boldsymbol{\mu}_{m}, \boldsymbol{\Sigma}_{m}, v_{m}\right\},(m=1,2, \cdots, M)
\end{aligned}
$$

where $\Psi=\left\{\pi_{\mathrm{m}}, \Psi_{\mathrm{m}}\right\}$ denotes the set of model parameters and $M$ the mixture number of densities in the model. In (4), each mixture component $p_{D}\left(x_{k} ; \mu_{m}, \Sigma_{m}, U_{m}\right)$ is the conditional probability density function (pdf.) of $x_{k}$ with the parameter $\Psi_{\mathrm{m}}$, which can be interpreted as the pdf. of those data points that form a corresponding cluster, say Cluster $m$, and $\pi \mathrm{m}$ is the proportion of data points that belong to Cluster $m$. In (5), $\Psi_{m}=\left\{\mu_{m}, \Sigma_{m}, U_{m}\right\}$ denotes the density of the t-distribution with mean $\mu_{m}$, covariance matrix $\Sigma_{m}$, and degree of freedom $U_{m}$.

As we know, ifu $>1, \mu_{m}$ is the mean of the population, and ifu $>2, \Sigma_{m}$ is the covariance matrix. The caseu $\rightarrow+\infty$ corresponds to a multivariate Gaussian distribution with mean $\mu_{m}$ and covariance matrix $\Sigma_{m}$, and whenu $=1$, this becomes multivariate Cauchy distribution. So the value of $u$ can tune the distribution, and t-distribution is more robust than Gaussian or Gamma distributions.

As aforementioned, a t-mixture model can describe the statistical characteristics of HRRPs more accurately than Gaussian model. This paper proposed that echoes of different types of cells are modeled as the t-distribution form.

\section{PARAMETER ESTIMATION}

When a statistical model is available, the classifier training problem is turned into a parameter estimation problem.

There are three sets of parameters to estimate: the values of $\pi_{\mathrm{m}}$, the component of the vectors $\Psi_{m}$ and the value of $M$. As we know, the more mixture components the $\mathrm{t}$ mixture distribution has, the more precise the estimated pdf. will be. However, too complex data representation model may result in overfitting and cannot guarantee good generalization performance. The estimation of $M$ is rather difficult, even though some techniques have been proposed. In this paper, we assume that $M$ is known as four.

\section{A. EM Algorithm}

In the EM framework, the data are viewed as incomplete, and the complete version is given by

$$
\mathrm{X}_{\mathrm{C}}=\left\{\mathrm{X}, Z_{1}, \cdots, Z_{N}\right\}
$$

where $X=\left\{x_{1}, x_{2}, \cdots x_{N}\right\}$ denotes the observed data set, and $\mathrm{Z}_{k}=\left(\mathrm{z}_{k 1}, \mathrm{Z}_{k 2}, \cdots \mathrm{Z}_{k M}\right)^{T}$ is the indicator variables $\mathrm{z}_{k m}=1$ if $x_{k}$ come from component $m$, zero otherwise, and $\mathrm{z}_{k}, k=1,2, \cdots N$ are independently and identically distributed according to a multinomial distribution taking $M$ mutually exclusive values with probability $\left\{\pi_{1}, \pi_{2}, \cdots \pi_{M}\right\}$. Regarding the indicator variables as missing data, a loglikelihood function can be written as

$$
\begin{aligned}
& \mathcal{L}\left(\boldsymbol{\Psi} \mid \mathrm{X}_{C}\right)=\sum_{k=1}^{N} \sum_{m=1}^{M} z_{k m} {\left[\ln p_{D}\left(\mathbf{x}_{k} ; \boldsymbol{\mu}_{m}, \boldsymbol{\Sigma}_{m}, v_{m}\right)\right.} \\
&\left.+\ln \pi_{m}\right]
\end{aligned}
$$

We notice that the multivariate t-distribution can be viewed as a weighted average multivariate Gaussian distribution with the weight Gamma distribution. Therefore, we introduce another set of "missing data" $W_{k}$, when $\mathrm{z}_{k m}=1$, $x_{k} \mid\left(w_{k}, \mathrm{z}_{k m}=1\right)$ is independently and identically distributed (IID), and $w_{k} \mid\left(\mathrm{z}_{k m}=1\right) \sim G\left(u_{m} / 2, \cup_{m} / 2\right),(k=1,2, \cdots N)$. 
Now the "complete data" set can be rewritten as corresponding loglikelihood function is

$$
\begin{gathered}
\mathrm{X}_{C}^{\prime}=\left\{\mathrm{X}, Z_{1}, \cdots, Z_{N}, \mathrm{~W}_{1}, \cdots, \mathrm{W}_{N}\right\} \\
\mathcal{L}\left(\boldsymbol{\Psi} \mid \mathrm{X}_{C}^{\prime}\right)=\sum_{k=1}^{N} \sum_{m=1}^{M} z_{k m}\left[\ln p\left(\mathbf{x}_{k} \mid \mathrm{w}_{k}, z_{k m}=1 ; \Psi\right)\right. \\
\left.+\ln p\left(\mathrm{~W}_{k} \mid z_{k m}=1 ; \Psi\right)+\ln p\left(z_{k m}=1 ; \Psi\right)\right]
\end{gathered}
$$

As shown by Peel [15] et al., the ML estimate of $\Psi$ can be achieved by iterating the following two steps

- E-Step: Given an input the $\mathbf{x}_{k}$, we fix $\Psi^{(t)}$ and cumulate $\mathrm{z}_{k m}{ }^{(t)}, \mathrm{W}_{k}{ }^{(t)}$ in (10), (11) respectively.

$$
\begin{gathered}
\hat{z}_{k m}^{(t)}=\frac{p\left(\mathrm{x}_{k} \mid \boldsymbol{\psi}_{m}^{(t)}\right) \pi_{m}^{(t)}}{p\left(\mathrm{x}_{k} ; \Psi^{(t)}\right)}=\frac{\pi_{m}^{(t)} p\left(\mathrm{x}_{k} \mid \boldsymbol{\psi}_{m}^{(t)}\right)}{\sum_{j=1}^{M} \pi_{j}^{(t)} p\left(\mathrm{x}_{k} \mid \boldsymbol{\psi}_{j}^{(t)}\right)} \\
\mathrm{W}_{k}^{(t)}=\frac{v_{m}^{(t)}+D}{\boldsymbol{v}_{m}^{(t)}+\delta\left(\mathrm{x}_{k}, \boldsymbol{\mu}_{m}^{(t)} ; \boldsymbol{\Sigma}_{m}^{(t)}\right)}
\end{gathered}
$$

$\delta\left(\mathrm{x}_{k}, \boldsymbol{\mu}_{m}^{(t)} ; \boldsymbol{\Sigma}_{m}^{(t)}\right)=\left(\mathrm{x}_{k}-\boldsymbol{\mu}_{m}^{(t)}\right)^{T}\left(\boldsymbol{\Sigma}_{m}^{(t)}\right)^{-1}\left(\mathrm{x}_{k}-\boldsymbol{\mu}_{m}^{(t)}\right)$

- $\quad$ M-Step: The mixing proportions $\pi_{m}$, are updated by the average of the posterior probabilities of component membership of the mixture. The estimates of $\boldsymbol{\mu}_{m}$, and $\boldsymbol{\Sigma}_{m}$ are also updated.

$$
\left\{\begin{array}{l}
\pi_{m}^{(t+1)}=\frac{1}{N} \sum_{k=1}^{N} p\left(m \mid \mathbf{x}_{k}, \Psi^{(t)}\right) \\
\boldsymbol{\mu}_{m}^{(t+1)}=\frac{\sum_{k=1}^{N} p\left(m \mid \mathbf{x}_{k}, \Psi^{(t)}\right) \mathbf{W}_{k}^{(t)} \mathbf{x}_{k}}{\sum_{k=1}^{N} p\left(m \mid \mathbf{x}_{k}, \Psi^{(t)}\right) \mathbf{W}_{k}^{(t)}} \\
\boldsymbol{\Sigma}_{m}^{(t+1)}=\frac{\sum_{k=1}^{N} p\left(m \mid \mathbf{x}_{k}, \Psi^{(t)}\right) \mathbb{W}_{k}^{(t)}\left(\mathbf{x}_{k}-\boldsymbol{\mu}_{m}^{(t+1)}\right)\left(\mathbf{x}_{k}-\boldsymbol{\mu}_{m}^{(t+1)}\right)^{T}}{N \pi_{m}^{(t+1)}}
\end{array}\right.
$$

The above two steps are repeatedly implemented for each input until $\Psi$ converges.

\section{B. A modified EM Algorithm}

Initialization of EM is a critical issue because EM converges to a local maximum of the likelihood function: the final estimate depends on the initialization. There are several initialization algorithms, such as HCM, LBG (Linde-Buzo-Gray) and fuzzy c-mean (FCM) clustering method.

Fuzzy clustering methods allow objects to belong to several clusters simultaneously, with different degrees of membership. The data set $\mathrm{X}$ is thus partitioned intov fuzzy subsets. In many real situations, fuzzy clustering is more natural than hard clustering, as objects on the boundaries between several classes are not forced to fully belong to one of the classes, but rather are assigned membership degrees between 0 and 1 indicating their partial memberships. In this case, we used the FCM clustering method for initialization of TMM in each cluster and then the two steps (E-step \& M-step) are applied.

The fuzzy K-means clustering algorithm is based on the minimization of the following objective function

$$
J(\mathrm{U}, \mathrm{V})=\sum_{i=1}^{N} \sum_{j=1}^{c} u_{i j}^{b} d^{2}\left(\mathbf{x}_{i}, \mathrm{v}_{j}\right)
$$

with

$$
\begin{aligned}
& u_{i j} \in[0,1], \quad(i=1,2, \cdots, N ; j=1,2, \cdots, c) \\
& \sum_{j=1}^{c} u_{i j}=1, \quad \forall i=1,2, \cdots, N \\
& 0<\sum_{i=1}^{N} u_{i j}<N, \quad(j=1,2, \cdots, c)
\end{aligned}
$$

where $\mathbf{U}=\left(u_{i j}\right)_{N{ }{ }_{c}}$ denotes the fuzzy cluster matrix, and $u_{i j}$ is the degree of membership of $x_{j}$ in the $i^{t h}$ cluster. The fuzzy $\mathrm{C}$-means clustering algorithm is based on the minimization of the following objective function

$$
\begin{aligned}
J(\mathbf{U}, \mathbf{V})= & \sum_{i=1}^{N} \sum_{j=1}^{c} u_{i j}^{b} d\left(\mathbf{x}_{i}, \mathbf{v}_{j}\right) \\
& -\sum_{i=1}^{N} \lambda_{i}\left(\sum_{j=1}^{c} u_{i j}-1\right)
\end{aligned}
$$

The fuzzy set will be determined by using an iterative method where $J(\mathbf{U}, \mathbf{V})$ is successively minimized with respect to fuzzy membership $u_{i j}$ and cluster centroid $v_{j}$.

Now we construct the modified EM algorithm for multivariate TMM.

1) Initialization. Using FCM method, we initialize the parameters of TMM, including initializing the $M, \pi{ }_{\mathrm{m}}, \mu$

${ }_{m}$ and $\boldsymbol{\Sigma}{ }_{m}$. Then, at each time step $t$, we implement the

following two steps of EM algorithm, as aforementioned

2) E-step;

3) M-step.

So far, we can build a TMM statistical model to represent the statistical characteristics of target HRRP.

\section{EXPERIMENTAL RESULT}

The main procedure of radar HRRP target recognition based on the modified algorithm for the proposed statistical model will be described in the following.

\section{A. Data Description}

This results presented in this paper are based on simulated airplane data [6]. The time-domain data of three groups of targets, namely, a Large-sized, a Middle-sized and a Small-sized target groups, consisting of 8 complex targets 
(aircrafts: $\mathrm{T}_{1}, \mathrm{~T}_{2}, \mathrm{~T}_{3}, \mathrm{~T}_{4}, \mathrm{~T}_{5}, \mathrm{~T}_{6}$, and cruise missiles $\mathrm{T}_{7}, \mathrm{~T}_{8}$ ) are simulated using the Radar Target Backscattering Simulation software [6]. The simulation parameters are as follows: The central radar frequency is $10.0 \mathrm{GHz}$ with a chirped pulse waveform with a bandwidth of $1.0 \mathrm{GHz}$. The signatures for each target are sampled over the azimuthal range of 0 to $180^{\circ}$ from nose-on, with an azimuthal interval of $0.05^{\circ}$. The antenna polarization is $\mathrm{H}-\mathrm{H}$.

In order to compare the identification rates in different aspect area, we select four sets, namely, $6^{\circ}, 30^{\circ}, 60^{\circ}$ and 90 ${ }^{\circ}$ as the recognition sets. Our target recognition is of 8 targets in each aspect set. We divide the simulated data into two subsets: the odd series are used for training, and the even series are used for testing. We use training data from different target-aspect angles to establish different frame models.

Some preprocessing needs to be applied to the range profiles (both training and test) to ensure that the targets are consistently aligned. In this paper, this is done by time-shift compensated using the slide correlation processing.

To investigate the noise performance of the proposed identification method, Gaussian noise are generated and added to the simulated target signature data with certain signal-to-noise ratio $(S N R)$ defined by

$$
S N R=10 \times \log _{10}\left(\frac{E_{s}}{\sigma_{n}^{2}}\right)
$$

where $E_{s}$ is the total energy of the frequency-domain data, $\sigma_{n}^{2}$ is the variance of the additive noise.

\section{B. Recognition Experiments}

Here is the identification result of 8 radar targets. Table I and Table II show the correct recognition rates of TMM with the modified EM algorithm in different aspect sets, and with $S N R=15 \mathrm{~dB}$ and $S N R=20 \mathrm{~dB}$, respectively. Obviously, the correct recognition rates $\mathrm{P}_{\mathrm{d}}$ increase as the aspect set gets smaller when the $S N R$ is fixed. It can also be seen that, for fixed aspect set, the correct recognition rates increase with higher $S N R$. When $S N R=15 \mathrm{~dB}$, in $6^{\circ}$ aspect set, the average $\mathrm{P}_{\mathrm{d}}$ of 8 targets is $97.88 \%$; whereas, $S N R=20 \mathrm{~dB}$, in $90^{\circ}$ aspect set, the average $\mathrm{P}_{\mathrm{d}}$ of 8 targets is $65.56 \%$.

TABLE I: THE CORRECT RECOGNITION RATES OF TMM WITH SNR=15DB $(\%)$

\begin{tabular}{|c|l|l|l|l|}
\hline \multirow{2}{*}{ Target } & \multicolumn{4}{|c|}{ Aspect Set (deg) } \\
\cline { 2 - 5 } & \multicolumn{6}{|c|}{$\mathbf{6}$} & \multicolumn{1}{|c|}{$\mathbf{3 0}$} & \multicolumn{6}{c|}{$\mathbf{9 0}$} & \multicolumn{1}{c|}{$\mathbf{0}$} \\
\hline $\mathrm{T}_{1}$ & 100 & 86.5 & 69.25 & 61.5 \\
\hline $\mathrm{T}_{2}$ & 100 & 97.75 & 90.5 & 91.5 \\
\hline $\mathrm{T}_{3}$ & 100 & 100 & 79 & 69.25 \\
\hline $\mathrm{T}_{4}$ & 100 & 84.25 & 74 & 55.5 \\
\hline $\mathrm{T}_{5}$ & 100 & 82 & 53.25 & 40.25 \\
\hline $\mathrm{T}_{6}$ & 97.75 & 82.5 & 62.5 & 58.25 \\
\hline $\mathrm{T}_{7}$ & 93.25 & 30 & 18.25 & 13 \\
\hline $\mathrm{T}_{8}$ & 92 & 29.5 & 17.5 & 15.5 \\
\hline $\begin{array}{c}\text { Average } \\
\text { recognition rate } \mathrm{P}_{\mathrm{d}}\end{array}$ & 97.88 & 74.06 & 58.03 & 50.59 \\
\hline
\end{tabular}

The average recognition rates of TMM, Gaussian mixture model and template matching method under the maximum correlation coefficient criterion (MCC) [1] versus the SNR $\left(6^{\circ}\right.$ aspect set) are shown in Fig. 1. From Fig. 1 it is clearly seen that: (1) statistical models have better recognition performances than the nonstatistical methods; (2) the average recognition rate of the TMM, with $S N R=15 \mathrm{~dB}$, is approximately 4 percentage points larger than those of Gaussian mixture model.

TABLE II: THE CORRECT RECOGNITION RATES OF TMM WITH SNR=20DB (\%)

\begin{tabular}{|c|l|l|l|l|}
\hline \multirow{2}{*}{ Target } & \multicolumn{4}{|c|}{ Aspect Set (deg) } \\
\cline { 2 - 5 } & $\mathbf{6}$ & $\mathbf{3 0}$ & $\mathbf{6 0}$ & $\mathbf{9 0}$ \\
\hline $\mathrm{T}_{1}$ & 100 & 100 & 89.25 & 80.5 \\
\hline $\mathrm{T}_{2}$ & 100 & 100 & 95.5 & 88.5 \\
\hline $\mathrm{T}_{3}$ & 100 & 100 & 97 & 83.5 \\
\hline $\mathrm{T}_{4}$ & 100 & 100 & 100 & 90.25 \\
\hline $\mathrm{T}_{5}$ & 100 & 100 & 75.75 & 60 \\
\hline $\mathrm{T}_{6}$ & 100 & 100 & 86.75 & 81 \\
\hline $\mathrm{T}_{7}$ & 100 & 40 & 23.25 & 19 \\
\hline $\mathrm{T}_{8}$ & 100 & 43.75 & 25.75 & 21.75 \\
\hline Average & 100 & 85.47 & 74.16 & 65.56 \\
\hline recognition rate $\mathrm{P}_{\mathrm{d}}$ & & & & \\
\hline
\end{tabular}

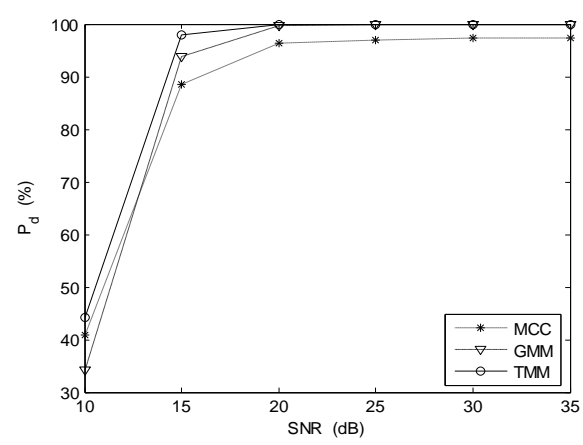

Fig. 1. The average recognition rates of MCC method, GMM and TMM versus the SNR.

\section{CONCLUSION}

We have developed a t-mixture model (TMM) approach to radar automatic target recognition, in which echoes of target are modeled by t-mixture distributions. Estimation for the parameters of TMM is achieved by the modified EM algorithm, i.e., fuzzy EM algorithm (FEM). Compared to the Gaussian mixture model, the t-distribution has wider tail than Gaussian distribution and has robustness in the presence of the observations that were polluted by noise. Experimental results for simulated data show that the proposed statistical model not only has better recognition performance but also is more robust to noises than Gaussian mixture model.

\section{ACKNOWLEDGMENT}

The authors are grateful for helpful comments from the referee and the editor that greatly improved the paper.

\section{REFERENCES}

[1] H. J. Li and S. H. Yang, "Using range profiles as features vectors to identify aerospace objects," IEEE Transactions on Antennas and Propagation, vol. 41, no. 3, pp. 261-268, 1993.

[2] R. A. Mitchell and R. Dewall, "Overview of high range resolution radar target identification," presented at the Automatic Target Recognition Working Group, Monterey, CA, 1994.

[3] Y. C. Lu and K. C. Chang, "A neural network approach for high resolution target classification," in Proc. SPIE, vol. 2484, pp. 558-566, 1995.

[4] K. T. Kim, D. K. Seo, and H. T. Kim, "Efficient radar target recognition using the MUSIC algorithm and invariant feature," IEEE Trans. Antennas Propag., vol. 50, no. 3, pp. 325-337, 2002. 
[5] J. Zwart, R. Heiden, S. Gelsema, and F. Groen, "Fast translation invariant classification of HRR range profiles in a zero phase representation," Inst. Elect. Eng. Proc.-Radar Sonar Navig., vol. 150, no. 6, pp. 411-418, 2003.

[6] Y. D. Shirman, Computer simulation of aerial target radar scattering, recognition, detection, and tracking, Boston-London: Artech House, 2002, ch. 3, pp. 100-115.

[7] C. Y. Wang and X.J. Xu, "Some new results of radar target identification using high resolution range profiles," IEEE Antennas and Propagation Society, AP-S International Symposium (Digest), pp. $122-125,2005$

[8] S. P. Jacobs, "Automatic target recognition using high-resolution radar range profiles," Ph.D. dissertation, Washington Univ., St. Louis, MO, 1999.

[9] D. Beckman and S. Frame, "Comparison of features from SAR and GMTI imagery of ground targets," in Proc. SPIE, vol. 5095, pp. 224-232, 2003

[10] K. Copsey and A. R.Webb, "Bayesian gamma mixture model approach to radar target recognition," IEEE Trans. Aerosp. Electron. Syst., vol. 39, no. 4, pp. 1201-1217, Oct. 2003.

[11] L. Du, H. W. Liu, Z. Bao, and J. Y. Zhang, "A two-distribution compounded statistical model for radar HRRP target recognition," IEEE Trans. Signal Process., vol. 54, no. 6, pp. 2226-2238, Jun. 2006.

[12] D. Peel and G. J. McLachlan, "Robust mixture modeling using the distribution," Stat. Comput. , vol. 10, pp. 339-348, 2000.

[13] S. Shoham, "Robust clustering by deterministic agglomeration EM of mixtures of multivariate t-distributions," Pattern Recognition, vol. 35 , no. 5, pp. 1127-1142, 2002.

[14] G. J. McLachlan and T. Krishnan, The EM algorithm and extensions, Wiley, New York, 1997, ch. 2, pp.100-131.
[15] G. J. McLachlan and D. Peel, Finite mixture models, Wiley, New York. ch. 3, 2000, pp.125-160.

[16] R. O. Duda, P. E. Hart, and D. G. Stork, Pattern classification, 2nd ed. New York: Wiley, ch.4, 2001, pp.180-185.

[17] L R. Gil-Pita , M. Rosa-Zurera, P. Jarabo-Amores, and F. Lo' pez Ferreras, "Statistical analysis of the zero-phase method for aligning noisy high-resolution radar signals," IET Radar Sonar Navigation, vol. 3 , no. 1 , pp. 62-69, 2009 .

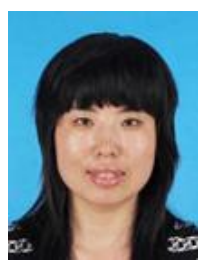

Caiyun Wang was born in Shanxi, China, on September 30, 1975. She graduated in 1996 with a B.S. degree and in 1999 with a M.S. degree. She received the Ph.D. degree in signal and information processing from Beihang University, Beijing, China, in 2008.

She is currently an associate professor with the School of Academy of Frontier Science, Nanjing University of Aeronautics and Astronautics (NUAA). Her major research interests are in the fields of radar automatic target recognition (RATA), radar signal processing, and adaptive signal processing, and pattern recognition

Jiling Xie was born in Shandong, China, on January 21, 1981. He received the Ph.D. degree in signal and information processing from Harbin Engineering University, Harbin, China, in 2008. His major research interests are in the fields of spatial spectrum estimation, radar target detection and signal processing. 